\title{
Comparison of NADH and NADPH Oxidase Activities in Granules Isolated from Human Polymorphonuclear
} Leukocytes with a Fluorometric Assay

\author{
David Iverson, Lawrence R. DeChatelet, John K. Spitznagel, and Patsy Wang \\ From The Department of Biochemistry, Bowman Gray School of Medicine, Winston-Salem, \\ North Carolina 27103, and The Department of Bacteriology and Immunology, University of North \\ Carolina Medical School, Chapel Hill, North Carolina 27514
}

\begin{abstract}
A B S T RA C T A fluorometric method for the determination of pyridine nucleotides has been adapted for use in studying the reduced pyridine nucleotide oxidases in human polymorphonuclear leukocytes. In the presence of strong base the oxidized forms of the pyridine nucleotides form a highly fluorescent product. The small amounts of $\mathrm{NAD}(\mathrm{P})$ formed by the oxidase reactions can be determined with great sensitivity. This method has been compared to the radioisotopic assay for NADPH oxidation. Both methods gave essentially the same results in terms of nanomoles NADP produced by control, resting, and phagocytizing samples.
\end{abstract}

Both NADPH and NADH oxidase activities were insensitive to cyanide. NADPH oxidation had a $\mathrm{pH}$ optimum of 5.5, while that for NADH appeared to be 6.0. Granules isolated from phagocytizing cells routinely showed more activity toward both substrates (two to threefold) than granules from resting cells. Both activities were located primarily in a granule fraction prepared by differential centrifugation. Oxidation of NADPH was routinely four to five times that of NADH at all except very high substrate levels. Measurable NADH oxidation was rarely seen below $0.80 \mathrm{mM}$ NADH, while NADPH oxidation was easily measurable at $0.20 \mathrm{mM}$. One patient with chronic granulomatous disease was studied. At low substrate levels, there was no activity toward either substrate in granules isolated from either resting or phagocytizing cells of this patient, while granules isolated from normal control cells showed substantial activity at these substrate levels.

Purification of the activities has been initiated with linear sucrose gradients. Both activities co-sediment to

Received for publication 19 July 1976 and in revised form 20 October 1976. a very dense region of the gradient, a region different from that in which membrane or azurophil granules usually equilibrate. The peak gradient fractions show a 10-30-fold increase in specific activity over comparable granule fractions.

These data suggest that the oxidase activities are associated with one enzyme that has different affinities for the two substrates and support the contention that the oxidation of NADPH is responsible for the metabolic burst accompanying phagocytosis in human PMNL.

\section{INTRODUCTION}

A reduced pyridine nucleotide oxidase is thought to be the enzyme that initiates the respiratory burst accompanying phagocytosis in polymorphonuclear leukocytes (PMNL). ${ }^{1}$ This enzyme catalyzes the reaction of the reduced nucleotide with molecular oxygen producing the oxidized nucleotide and hydrogen peroxide. There has been some controversy over the substrate specificity of the oxidase; some work has implicated an NADH oxidase (1-3) while other studies have suggested NADPH oxidase $(4,5)$ as the critical enzyme. Both oxidase reactions can directly explain the increases in oxygen consumption and $\mathrm{H}_{2} \mathrm{O}_{2}$ production that neutrophils exhibit upon phagocytosis. Both can be used to explain the increase in the hexose monophosphate shunt activity: NADPH oxidase can directly generate NADP, the rate limiting substance for the hexose monophosphate shunt activity; NADH oxidase might indirectly generate NADP by means of a transhydrogenase (6) or by the glutathione peroxidase system (7). Implicit in these arguments seems to be the

\footnotetext{
${ }^{1}$ Abbreviations used in this paper: CGD, chronic granulomatous disease; MPO, myeloperoxidase; PBS, phosphate buffered saline; PMNL, polymorphonuclear leukocytes.
} 
assumption that there are two oxidase enzymes, one that uses NADPH and another that uses NADH; this has not been rigorously demonstrated. Although it is possible that there are two separate enzymes, it is also possible that there is a single enzyme which can oxidize either reduced pyridine nucleotide.

Work on the oxidases in general has been plagued by the lack of a sensitive, specific assay. A typical assay involves measuring oxygen uptake with a Clark electrode. This method is fairly nonspecific as it would measure any and all metabolic activities of the cells requiring oxygen, not necessarily just the oxidase, and variable nonenzymatic rates of oxygen consumption are seen from day to day (8). In addition, the assay is not very sensitive, requiring relatively large, nonphysiological amounts of nucleotide to observe activity in human cells. A spectrophotometric method which measures a decrease in absorbance at $340 \mathrm{~nm}$ with time has also been used. This method, though specific, also suffers from a lack of sensitivity. Since the pyridine nucleotides absorb so strongly, it is necessary to use low concentrations of the substrate or a $1-\mathrm{mm}$ path length to insure that the measurement is kept within the linear range of the spectrophotometer according to Beer's law. Such a concentration is lower than optimal for measuring the enzyme in subcellular fractions of human PMNL. Also, regardless of what concentration is used, one is forced by this method to measure a relatively small decrease in a relatively large initial absorbance reading. This is not a very accurate procedure as instrument noise becomes more and more important.

In 1975, DeChatelet and his co-workers published an isotopic procedure for assaying NADPH oxidase (8). This assay eliminated the problems of the others by being both absolutely specific and very sensitive. It measures the amount of NADP formed by coupling the oxidase reaction to the 6-phosphogluconic dehydrogenase reaction in a two-step incubation procedure. The major problem with this assay is its inability to measure the oxidation of NADH due to the absolute specificity of the dehydrogenase enzyme for NADP and thus its inability to answer the question of which oxidase is the important one in the respiratory burst of the human neutrophil.

Lowry et al. (9), expanding on the work of Kaplan et al. (10), devised a very sensitive assay system for the pyridine nucleotides relying on the fact that the reduced forms are destroyed by acid, while the oxidized forms are destroyed by base. Further, when the oxidized form of either nucleotide is incubated in strong base (i.e., $6 \mathrm{~N}$ ) the destruction yields a highly fluorescent product. Such a system allows measurement of a small amount of $\mathrm{NAD}(\mathrm{P})$ that is formed in the presence of a relatively large amount of $\mathrm{NAD}(\mathrm{P}) \mathrm{H}$ in a typical oxidase assay. In addition, nanomolar amounts of the nucleotides can be measured and the assay has a 2,000-fold useful range (9). These factors make this system a very powerful one, capable of dealing with most questions related to the oxidase problem.

\section{METHODS}

Preparation of crude granule fraction. Venous blood was obtained from normal, healthy volunteers. The erythrocytes were sedimented with plasmagel (HTI Corp., Buffalo, N. Y.) as previously described (8). The leukocytes were collected by centrifugation at $160 \mathrm{~g}$ for $8 \mathrm{~min}$ at $4^{\circ} \mathrm{C}$, washed once with Dulbecco's phosphate buffered saline (PBS), and contaminating erythrocytes removed by hypotonic lysis. The leukocytes were suspended to a final concentration of $1.5 \times 10^{8}$ $\mathrm{PMNL} / \mathrm{ml}$. Total and differential counts were performed. These preparations routinely yielded 70-80\% neutrophils, $1-7 \%$ eosinophils, and 10-20\% lymphocytes and monocytes.

Zymosan (ICN, Nutritional Biochemicals Division, International Chemical \& Nuclear Corp., Cleveland, Ohio) was suspended in PBS at a concentration of $50 \mathrm{mg} / \mathrm{ml}$. $2 \mathrm{vol}$ of human pooled serum was added to 1 vol of the zymosan suspension and incubated at $37^{\circ} \mathrm{C}$ for $30 \mathrm{~min}$. At the end of the incubation, the opsonized zymosan was sedimented by centrifugation at $17,000 \mathrm{~g}$ for $10 \mathrm{~min}$. The supernate was discarded and the zymosan was resuspended in $3 \mathrm{vol}$ of PBS.

$1 \mathrm{ml}$ of cell suspension was incubated with $2 \mathrm{ml}$ of PBS to give resting cells or with $2 \mathrm{ml}$ of opsonized zymosan to give phagocytizing cells. The PBS and zymosan were preincubated at $37^{\circ} \mathrm{C}$ for $5 \mathrm{~min}$, the cells were added, and the incubation continued for another $3 \mathrm{~min}$. At the end of this time $3.0 \mathrm{ml}$ of cold $0.68 \mathrm{M}$ sucrose was added and the cells were put immediately on ice. They were then homogenized to greater than $90 \%$ breakage in a Potter-Elvehjem homogenizer with a motor-driven Teflon pestle run at $12,000 \mathrm{rpm}$ (Tri-R Stirrer model S-63C operated at maximum speed; Tri-R Instruments, Inc., Jamaica, N.Y.). The whole homogenate was centrifuged at $500 \mathrm{~g}$ to remove unbroken cells, large debris, and zymosan particles. The $500 \mathrm{~g}$ supernate was then centrifuged at $27,000 \mathrm{~g}$, and the resulting granule pellet was resuspended in $0.34 \mathrm{M}$ sucrose with a Dounce homogenizer. The procedure of Lowry et al. was used to estimate protein (11). The granule fractions generally had greater than $1.0 \mathrm{mg} / \mathrm{ml}$ protein. For ease in comparing day-today results with different preparations, all preparations were routinely diluted to $1 \mathrm{mg} / \mathrm{ml}$ with $0.34 \mathrm{M}$ sucrose and $0.10-\mathrm{ml}$ samples were used for assay in the $1-\mathrm{ml}$ incubations.

Isopycnic centrifugation of PMNL granules. Venous blood was obtained, the erythrocytes sedimented, and the leukocytes collected as before. After the initial collection of the leukocytes, the cells were suspended in PBS to approximately $10^{7}$ cells $/ \mathrm{ml}$. $4 \mathrm{ml}$ of this solution was layered on $3 \mathrm{ml}$ of Ficoll-Hypaque solution (final Ficoll/Hypaque concentrations equal to $6.06 \% / 10.0 \%$ ) and centrifuged at $350 \mathrm{~g}$ for $35 \mathrm{~min}$ at $20^{\circ} \mathrm{C}$. This procedure effectively removed lymphocyte and monocyte contamination and routinely yielded preparations of 90-95\% neutrophils, 5-7\% eosinophils and basophils, and 1-3\% lymphocytes and monocytes. After Ficoll-Hypaque treatment the cell pellet was resuspended and washed in PBS. Hypotonic lysis removed erythrocyte contamination, and the PMNL were suspended to a final concentration of $1.5 \times 10^{8} / \mathrm{ml}$. The zymosan preparation and cell incubation were performed as described above.

At the end of the 3-min incubation the cell suspension was centrifuged and the cell pellet resuspended to a concentration of $3 \times 10^{8} \mathrm{cell} \mathrm{s} / \mathrm{ml}$ in $0.34 \mathrm{M}$ sucrose. This was done to facilitate 


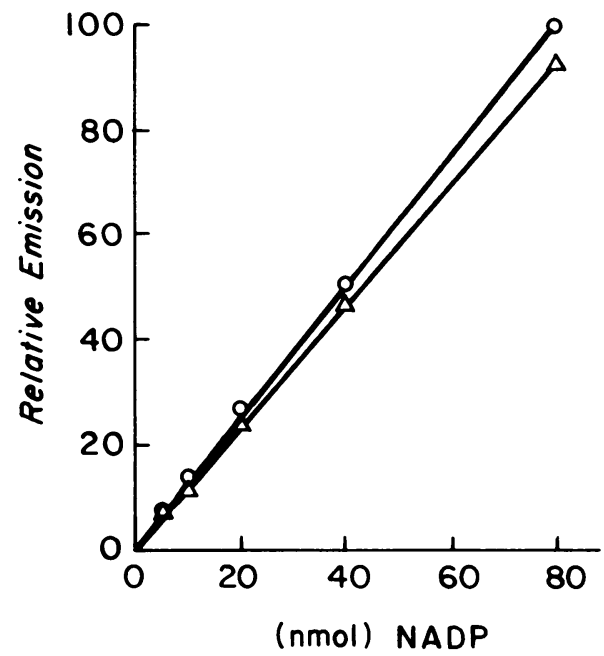

FIGURE 1 Effect of excess NADPH on standard curve. The circles represent standards without excess NADPH, the triangles represent standards with $200 \mathrm{nmol}$ NADPH. The horizontal axis denotes concentrations in terms of nmol NADP added to the $1-\mathrm{ml}$ incubation volume. The vertical axis is in arbitrary units: meter readings of the fluorometer expressed as percentages of the highest meter reading. Values represent means of triplicate determinations.

breaking the cells with the relatively low-speed motor $(1,900 \mathrm{rpm})$ used for homogenizing these preparations and to provide a volume small enough to load on the gradient. The cells were homogenized to $80 \%$ breakage in a PotterElvehjem homogenizer. The whole homogenate was diluted twofold with $0.34 \mathrm{M}$ sucrose and a sample was saved to determine recovery of the various enzyme markers from the gradient. The homogenate was centrifuged at $500 \mathrm{~g}$ as before, and samples of the $500-g$ supernate and resuspended pellet were saved for enzyme analysis. Oxidase activity cannot be measured in the 500-g supernate (probably due to an unidentified inhibitor), so to determine its recovery from the gradient, another sample of the 500-g supernate was centrifuged at $27,000 \mathrm{~g}$ to obtain a crude granule pellet. This was resuspended in $0.34 \mathrm{M}$ sucrose and used to determine the theoretical amount of oxidase activity put on the gradient. The remaining $500 \mathrm{~g}$ supernate was then made $25 \%$ in sucrose $(\mathrm{wt} / \mathrm{wt}$ ) and layered on a $50-\mathrm{ml}$ continuous sucrose gradient of $30-53 \%$ sucrose (wt/wt). The loading vol was $3-5 \mathrm{ml}$. The gradients were centrifuged at $21^{\circ} \mathrm{C}$ in a Beckman SW 25.2 rotor (Beckman Instruments, Inc., Cedar Grove, N. J.) $\left(R_{\max }=15.3\right.$ $\mathrm{cm}$ and $R_{\min }=6.4 \mathrm{~cm}$ ) at $2,000 \mathrm{rpm}$ for $15 \mathrm{~min}$ and then at $21,000 \mathrm{rpm}$ for $2 \mathrm{~h}$ where $\int_{0}^{t} \mathrm{w}^{2} \mathrm{dt}=3.7 \times 10^{10} \mathrm{~s}^{-1}(12)$. At the end of this time they were fractionated into $1-\mathrm{ml}$ fractions by piercing the tubes and pumping $60 \%$ sucrose from the bottom. The turbidity profiles of the gradients were obtained by measuring the absorbance at $450 \mathrm{~nm}$ of each fraction, and the percent sucrose was determined with a refractometer. To remove the sucrose, the fractions were either dialyzed against PBS overnight, or pooled, diluted with PBS, centrifuged at 27,000 $\mathrm{g}$ for $30 \mathrm{~min}$, and the pellets resuspended in PBS.

Enzyme assays. Protein in the granule fractions was estimated by the method of Lowry et al. (11) with bovine serum albumin and lysozyme as standards. Gradient protein was estimated with fluorescamine (13) with bovine serum albumin as the standard. Lactoferrin and myeloperoxidase were determined by single radial immunodiffusion (12).
Lysozyme was determined by the method of Osserman et al. (14). Nonspecific peroxidase was determined with odianisidine (15).

The oxidases were assayed in a two-step procedure. The first step had a $1-\mathrm{ml}$ incubation vol containing $0.1 \mathrm{M}$ potassium phosphate buffer ( $\mathrm{pH} 5.5$ for NADPH, pH 6.0 for $\mathrm{NADH}), 2.0 \mathrm{mM} \mathrm{KCN}$, and $0.1 \mathrm{mg}$ protein. Preliminary experiments demonstrated that $2.0 \mathrm{mM} \mathrm{KCN}$ did not inhibit either oxidase activity. Because of a significant degree of spontaneous or nonenzymatic oxidation of the reduced pyridine nucleotides, it was necessary to run controls in all experiments in which the protein was omitted from the incubation mixture. The reaction was started by adding the appropriate amount of $\mathrm{NAD}(\mathrm{P}) \mathrm{H}$ and incubating for 30 or $60 \mathrm{~min}$. The incubation was stopped by the addition of $1 \mathrm{ml}$ of $0.4 \mathrm{~N} \mathrm{HClO}_{4}$, and the protein was removed by centrifugation. A $0.10-\mathrm{ml}$ sample of the resulting supernate was added to 0.15 ml of $10 \mathrm{~N} \mathrm{NaOH}$ (final $\mathrm{NaOH}$ concentration was $6 \mathrm{~N}$ ) and allowed to incubate for $1 \mathrm{~h}$ at room temperature. At the end of this time, $1.6 \mathrm{ml}$ of water was added to each sample. Fluorescence of the samples was determined in a Farrand model A-4 filter fluorometer (Farrand Optical Co., Inc., Valhalla, N. Y.) equipped with a $365-\mathrm{nm}$ primary filter and a 448-nm secondary filter. A standard curve was run in parallel with each experiment so that the relative fluorescence could be directly converted to nanomoles of product formed.

\section{RESULTS}

The first part of this work was designed to adapt the procedure of Lowry et al. for the fluorometric determination of pyridine nucleotides to use in the oxidase assay. Standard amounts $(5,10,20,40$, and so on to 160 $\mathrm{nmol} / \mathrm{ml}$ ) of NAD or NADP were assayed as described in Methods. The assay was linear up to an initial concentration of $160 \mathrm{nmol} / \mathrm{ml} \mathrm{NAD}(\mathrm{P})$ (data not shown). Lowry's original procedure used $0.4 \mathrm{~N} \mathrm{HCl}$ to eliminate excess $\mathrm{NAD}(\mathrm{P}) \mathrm{H}$; we compared $0.4 \mathrm{~N} \mathrm{HCl}$ with $0.4 \mathrm{~N}$ $\mathrm{HClO}_{4}$ and obtained identical standard curves with both (data not shown). Consequently, $0.4 \mathrm{~N} \mathrm{HClO}_{4}$ was used to stop the reaction because it both precipitated protein and destroyed excess $\mathrm{NAD}(\mathrm{P}) \mathrm{H}$.

It was important to know whether or not small amounts of NAD $(\mathrm{P})$ could be detected in the presence of a large excess of $\mathrm{NAD}(\mathrm{P}) \mathrm{H}$. Presumably, the use of acid to stop the reaction would destroy the excess reduced forms but would not affect the acid-stable oxidized forms. Consequently, standard curves were run as above with and without excess $\mathrm{NAD}(\mathrm{P}) \mathrm{H}$, as illustrated in Fig. 1. The presence of $200 \mathrm{nmol}$ of NADPH did not significantly affect the standard curve. A similar experiment was performed with NAD in the presence and absence of NADH, yielding similar results.

Experiments were then performed on granule fractions isolated from resting and phagocytizing cells. Oxidation of both NADPH and NADH was consistently greater in the granules isolated from phagocytizing cells. To further validate the fluorometric assay procedure, NADPH oxidase was measured both with this fluorometric procedure and the isotopic pro- 

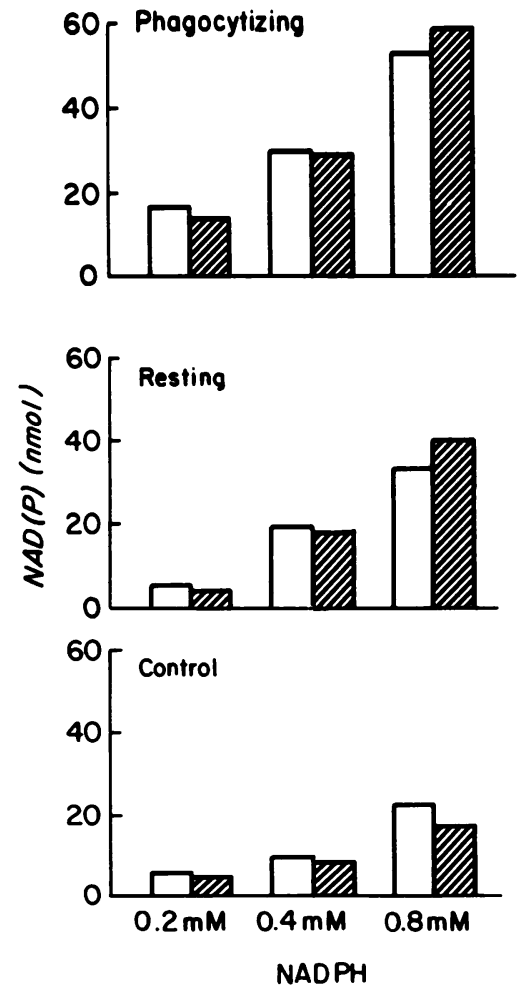

FIGURE 2 Comparison of the fluorometric and isotopic assays for NADPH oxidase. The open bars show the results obtained from the fluorometric method, the hatched bars represent the isotopic method. Results are shown for control (spontaneous oxidation) and for granule fractions obtained from resting and phagocytizing cells at three levels of substrate concentration and $0.1 \mathrm{mg}$ protein per assay. The vertical axis represents total NADP formed in the 1-ml assay volume in $30 \mathrm{~min}$. Values are means of triplicate determinations.

cedure developed by DeChatelet et al. (8). The results are shown in Fig. 2. Agreement between the two methods of assay was excellent; essentially the same activity was observed with each method for control, resting, and phagocytizing samples at three different levels of substrate.

With the assay system validated, we proceeded to characterize the oxidase activity in the neutrophil. We first examined oxidase activity in various subcellular fractions: the 500-g pellet, the granule fraction, and the 27,000-g supernate. The results are illustrated in Fig. 3. The 27,000-g supernate had no activity toward either substrate up to $0.80 \mathrm{mM} \mathrm{NAD}(\mathrm{P}) \mathrm{H}$ concentration. The 500-g pellet showed no activity toward NADH, yet there was some activity with NADPH at all levels of substrate, being most apparent at high concentrations. The granule fraction showed the greatest activity. Activity toward NADH was seen clearly only at a high concentration of substrate (i.e., $0.80 \mathrm{mM}$ NADH), while NADPH oxidase was easily measurable at all levels of substrate examined. This was a consistent observation: activity of NADPH oxidase was seen easily at $0.20 \mathrm{mM}$ substrate in the granule fraction, while activity of NADH oxidase was rarely observed below $0.80 \mathrm{mM}$ NADH. The activity toward NADH could not be explained by the presence of a transhydrogenase acting upon endogenous NADP. Although there is measurable transhydrogenase activity in the whole homogenate, no activity is seen in the granule fraction under the conditions employed in the oxidase assay (unpublished experiments). In another experiment the whole homogenate was examined for oxidase activity, a small amount was found only at $0.80 \mathrm{mM} \mathrm{NADPH}$, none at all for NADH (data not shown).

Fig. 4 illustrates the effect of incubation time on oxidation of both NADH and NADPH by the granule

NADPH OXIDASE

NADH OXIDASE
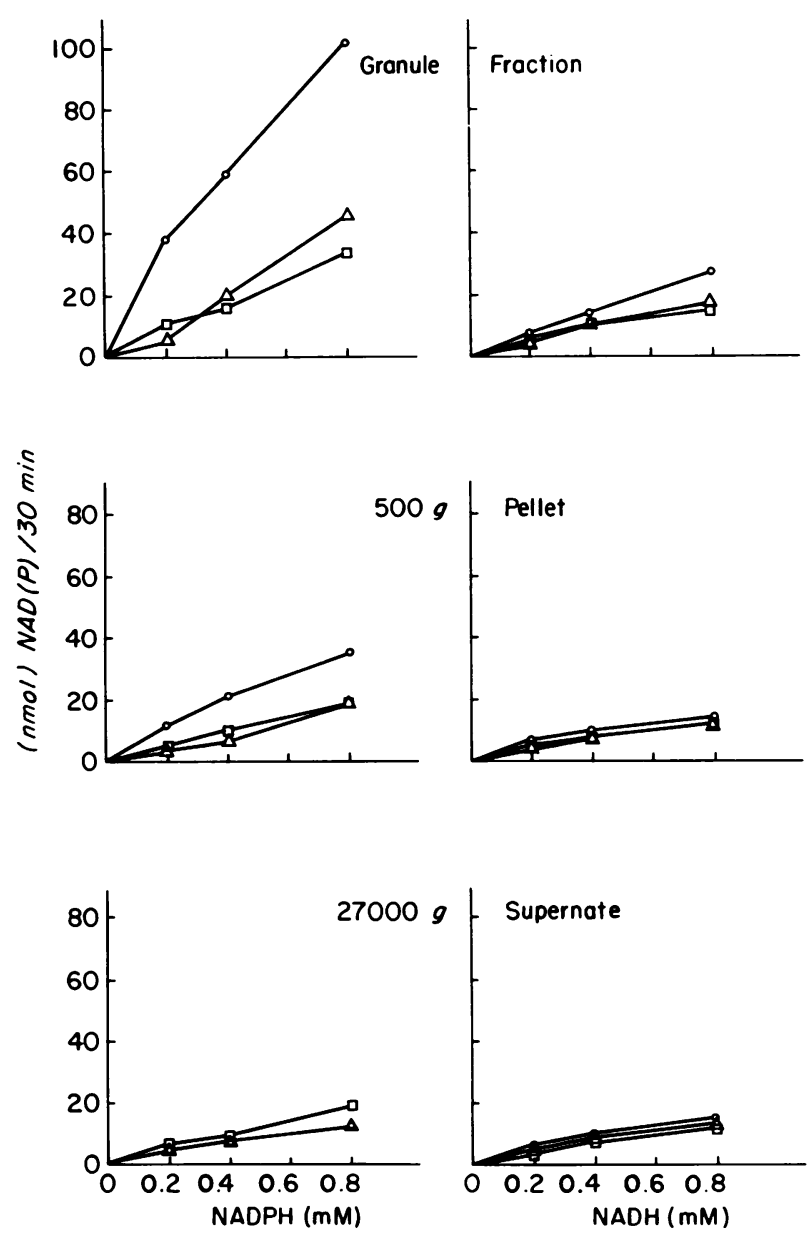

FIGURE 3 Oxidase activity in various fractions. The squares represent the spontaneous oxidation of $\mathrm{NAD}(\mathrm{P}) \mathrm{H}$ under assay conditions. The triangles represent fractions isolated from resting cells, the circles fractions from phagocytizing cells. $0.2 \mathrm{mg}$ protein was used in assaying the $500 \mathrm{~g}$ pellet and the 27,000-g supernate; $0.1 \mathrm{mg}$ was used for the granule fractions. Values represent means of triplicate determinations. 


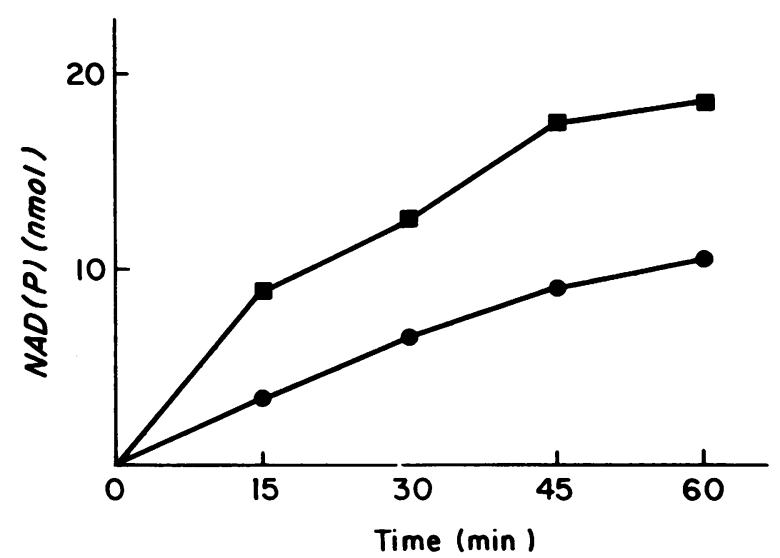

FIGURE 4. Effect of time of incubation on oxidase activity. The squares show results from NADPH oxidase activity; the circles show NADH oxidase activity. $0.1 \mathrm{mg}$ granule protein isolated from phagocytizing cells was used with $0.20 \mathrm{mM}$ NADPH or $0.80 \mathrm{mM}$ NADH. The vertical axis gives total number of nmol of $\mathrm{NAD}(\mathrm{P})$ formed in the 1-ml incubation volume. Values are means of triplicate determinations corrected for spontaneous oxidation.

fraction from phagocytizing cells. Oxidation increased progressively up to at least $60 \mathrm{~min}$ in a fairly linear fashion. Fig. 5 shows a $\mathrm{pH}$ curve for both activities; oxidation of NADPH had an apparent optimum $\mathrm{pH}$ of 5.5 as reported earlier (8); NADH oxidation had an apparent optimum of 6.0. It should be noted in these two figures that there was a substantial difference in the activities toward the two substrates reflected in the fact that the concentration of NADPH in the assay mixture was $0.20 \mathrm{mM}$ while that of NADH was four times higher $(0.80 \mathrm{mM})$. The effect of protein concentration was likewise deter-

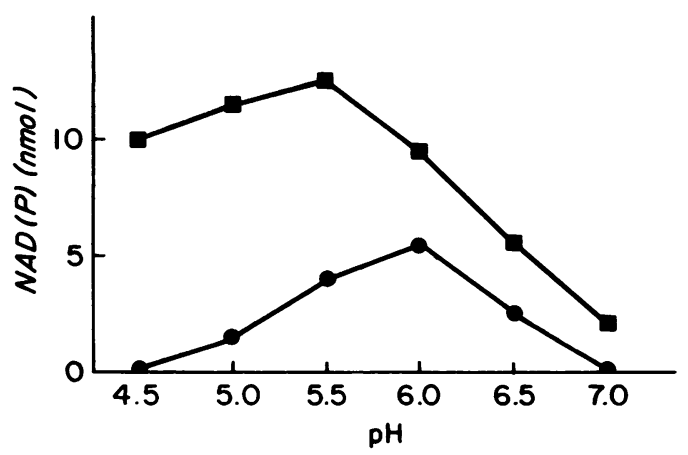

Figure 5 Effect of $\mathrm{pH}$ on oxidase activities. The squares show NADPH oxidase activity with $0.20 \mathrm{mM}$ NADPH; the circles show NADH oxidase activity at $0.80 \mathrm{mM}$ NADH. 0.1 mg granule protein isolated from phagocytizing cells was used. The horizontal axis represents the $\mathrm{pH}$ of the 0.1-M potassium phosphate buffers used. The vertical axis represents total nano moles $\mathrm{NAD}(\mathrm{P})$ formed in the 1-ml incubation volume in $30 \mathrm{~min}$. Values are means of triplicate determinations corrected for spontaneous oxidation. mined for both substrates. Both activities were linear up to approximately $0.2 \mathrm{mg}$ protein per assay when the incubation time was $30 \mathrm{~min}$ (data not shown). Table I shows the compilation of data from several experiments assayed at $0.20 \mathrm{mM}$ NADPH and $0.80 \mathrm{mM}$ $\mathrm{NADH}$. As can be seen, the phagocytizing samples showed significantly more activity than either control or resting samples. Also, in spite of the fourfold substrate concentration difference, there is more oxidation of NADPH than NADH in the phagocytizing samples.

Chronic granulomatous disease (CGD) is a childhood syndrome in which the PMNL fail to kill invading bacteria, and they do not show a normal increase in metabolic activity with phagocytosis (16). A defect in the oxidase could explain that observation and, in fact, previous reports showed there was no oxidation of NADPH with granules from patients with $\operatorname{CGD}(5,17)$. Fig. 6 shows the effect of substrate concentration at three levels of NADPH and NADH on the oxidase activities of granules obtained from a patient with CGD and granules from normal cells prepared in parallel. The granules from the patient with CGD showed no significant activity toward NADPH at the substrate levels examined. The normal control showed activity at all levels with resting activity becoming equal to phagocytizing activity at and above $0.80 \mathrm{mM}$. The same pattern holds for NADH; again no activity was observed with granules isolated from cells of the patient with CGD while activity was observed with granules isolated from normal cells at 0.40 and $0.80 \mathrm{mM}$ NADH. Granules from CGD cells did show some activity toward both substrates, but only at very high substrate levels (6-12 $\mathrm{mM} \mathrm{NAD(P)H)} \mathrm{and}$ no difference was seen between resting and phagocytizing samples (data not shown). The control cells used in this experiment were not typical of other

TABLE I

$N A D(P) H$ Oxidation in Control, Resting, and Phagocytizing Samples

\begin{tabular}{lcrr}
\hline $\begin{array}{l}\text { NAD(P)H } \\
\text { oxidation }\end{array}$ & Control & Resting & Phagocytizing \\
\hline nmol/30 min & & & \\
NADP & $8.2 \pm 0.8(10)^{*}$ & $4.6 \pm 1.0(8)$ & $24.4 \pm 1.6(10)$ \\
NAD & $10.1 \pm 0.7(11)$ & $10.9 \pm 1.2(9)$ & $17.7 \pm 1.6(11)$ \\
\hline
\end{tabular}

Values are averages \pm SEM for the number of experiments. Values in each experiment were determined in triplicate. Assay concentration of NADPH was $0.20 \mathrm{mM}$, NADH concentration was $0.80 \mathrm{mM}$. Protein concentration was 0.1 $\mathrm{mg} / \mathrm{ml}$. Control is the spontaneous oxidation of $\mathrm{NAD}(\mathrm{P}) \mathrm{H}$ under assay conditions. Resting and phagocytizing represent granule fractions isolated from resting and phagocytizing cells.

* Number of experiments. 
routine preparations as the oxidase activities, especially from the resting cells, were unusually high (see Fig. 3 and Table I). This preparation contained $15 \%$ eosinophils, an unusually high percentage. Unpublished data from this laboratory showed that eosinophils have three to five times more oxidase activity than do neutrophils, consequently the elevated activity shown here may be at least partially due to the elevated eosinophil count. That, however, does not change the observation that the CGD cells showed no activity toward either substrate, even if they are compared to the data in Fig. 3 or Table I.

Because of the relatively higher activity in the eosinophil than the neutrophil, the possibility must be considered that the measured activities reside entirely in the eosinophilic contamination and not in the neutrophil itself. This does not seem to be the case as evidenced by the following facts: (1) in one preparation from a normal subject, the isolated cells contained only $1 \%$ eosinophils, yet the specific activity of NADPH oxidase in this preparation was $32.3 \mathrm{nmol} / 30$

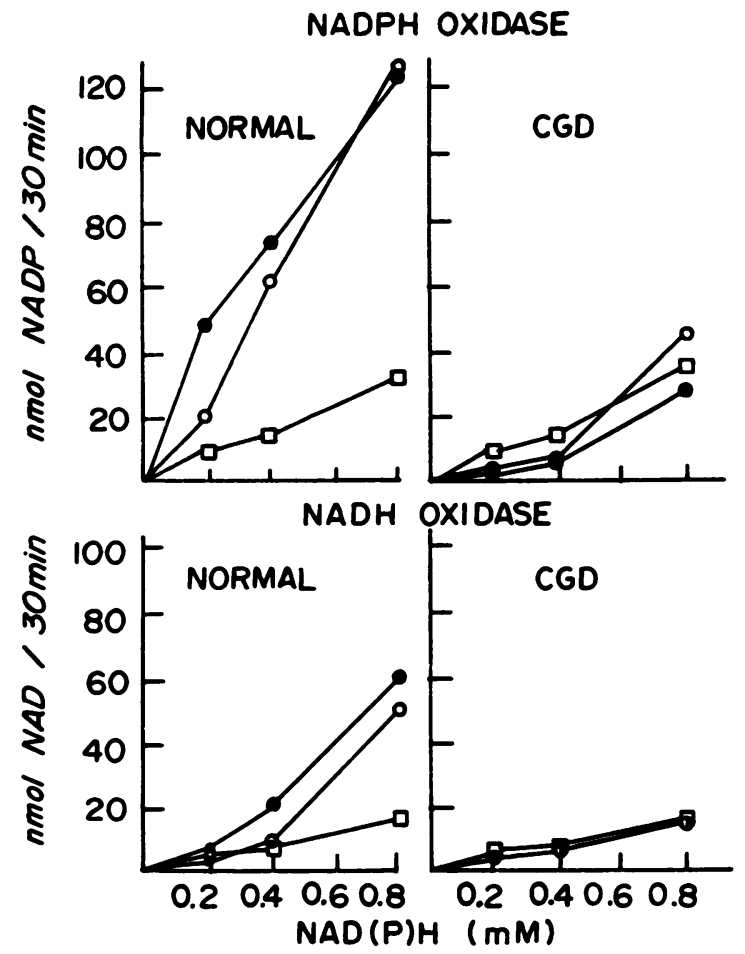

FIGURE 6 Effect of $\mathrm{NAD}(\mathrm{P}) \mathrm{H}$ concentration on $\mathrm{NAD}(\mathrm{P}) \mathrm{H}$ oxidase activities of granules isolated from CGD and normal PMNL. The top two panels show normal and CGD activity toward NADPH; the bottom panels show normal and CGD activity toward NADH. The squares show the spontaneous oxidation of $\mathrm{NAD}(\mathrm{P}) \mathrm{H}$ under assay conditions. The open circles represent granules obtained from resting cells; the closed circles represent granules from phagocytizing cells. Protein concentration was $0.1 \mathrm{mg}$. Each point represents the mean of triplicate determinations.

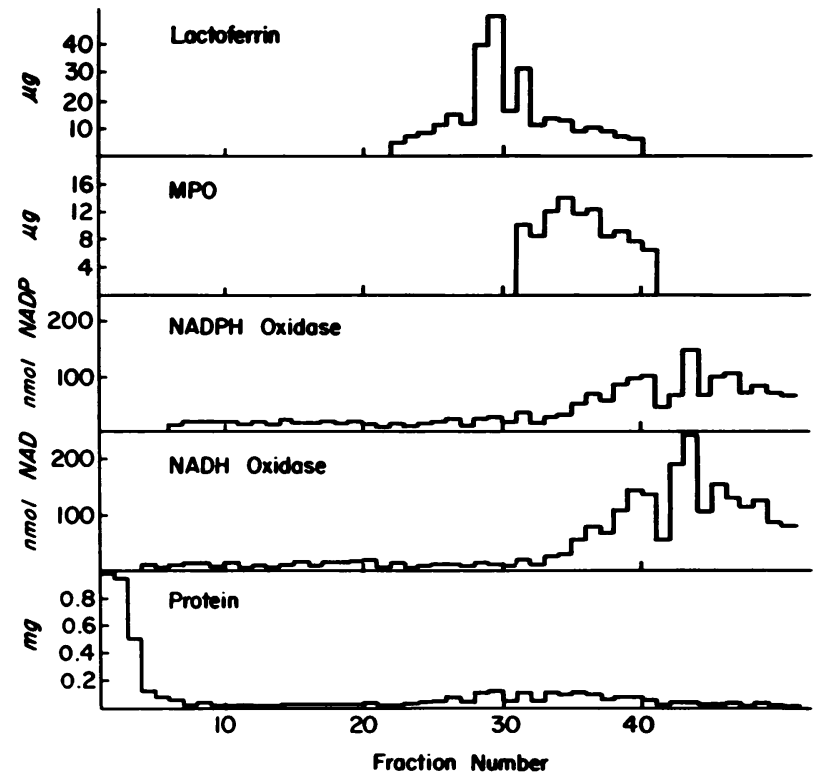

FIGURE 7 Location of NAD(P)H oxidase activity in a linear sucrose gradient. The samples of this gradient were dialyzed overnight against PBS. The vertical axes represent total activities found in each fraction. NADPH concentration was $0.20 \mathrm{mM}$, NADH $0.80 \mathrm{mM}$; incubation time was $60 \mathrm{~min}$.

$\mathrm{min} / 0.10 \mathrm{mg}$ protein. If anything, this is slightly higher than the average $\mathrm{sp}$ act of $24.4 \pm 1.6 \mathrm{nmol} / 30 \mathrm{~min} / 0.10$ mg protein reported in Table I. (2) We obtained a leukocyte preparation from a patient with pronounced eosinophilia. The specific activity of NADPH oxidase in a granule fraction isolated from phagocytizing cells containing $84 \%$ eosinophils was 3.2 times the activity seen in a paired preparation containing $82 \%$ neutrophils and only $3 \%$ eosinophils.

We next attempted to localize the oxidase activities on a linear sucrose gradient. Fig. 7 shows one such experiment. Lactoferrin and myeloperoxidase (MPO) indicate the locations of the specific and azurophil granules, respectively, in the gradient. The lactoferrin peak was at a density of approximately $1.18 \mathrm{~g} /$ cubic centimeter, the MPO peak at $1.22 \mathrm{~g} /$ cubic centimeter, while both oxidase activities appeared to migrate further into the gradient, with the peak activity at a density of approximately $1.24 \mathrm{~g} /$ cubic centimeter.

Fig. 8 shows another preparation in which the whole homogenate was divided in two parts; one part was initially centrifuged at $126 \mathrm{~g}$ while the second was centrifuged at $500 \mathrm{~g}$. The resulting low-speed supernates were then loaded on separate sucrose gradients. This was done to determine if the oxidase activities were due to eosinophil granules. Since eosinophil granules have a greater density than neutrophil granules, they sediment lower in the gradient (12) and this might have explained the very dense localization of the oxidase activities. It was known that many of the 


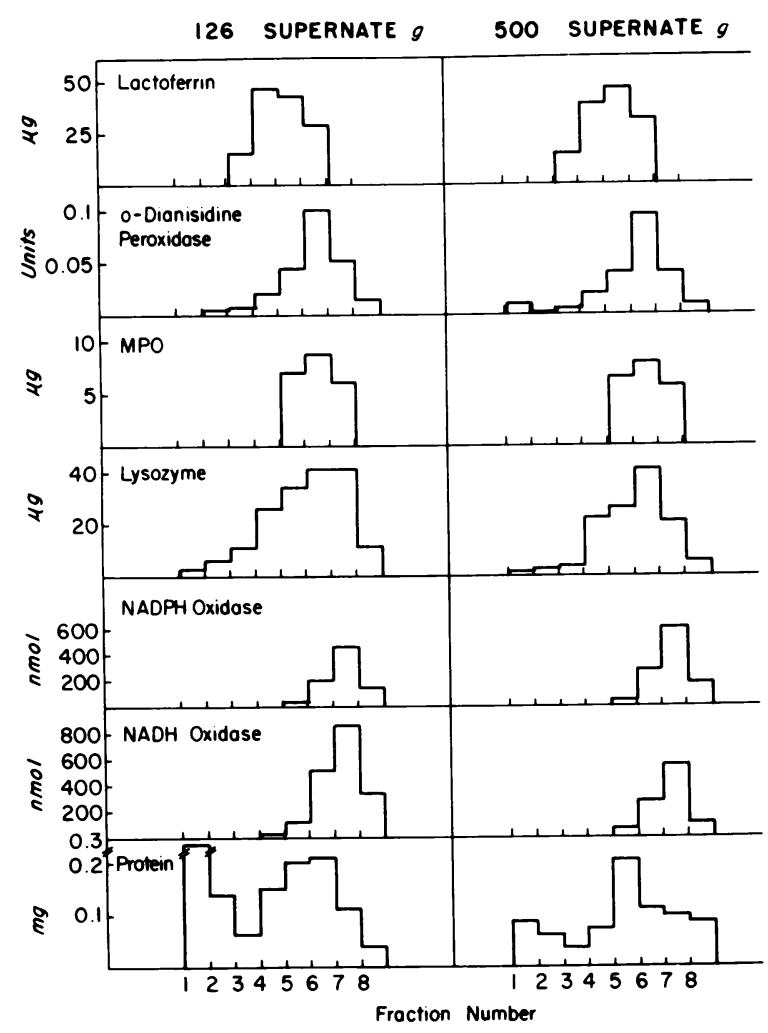

FIGURE 8 Location of $\mathrm{NAD}(\mathrm{P}) \mathrm{H}$ oxidase activity in a linear sucrose gradient. The samples of the gradient were pooled and centrifuged. The vertical axes represent total activity in each fraction. NADPH concentration was $0.20 \mathrm{mM}$, NADH $0.80 \mathrm{mM}$; incubation time was $60 \mathrm{~min}$.

eosinophil granules sediment at $500 \mathrm{~g}$ (18); consequently the whole homogenate in this experiment was given the two low-speed centrifugations to see if there would be a difference in the oxidase activities of the two gradients. No such distinction was detected. In this experiment the fractions were pooled, centrifuged, and resuspended. The disadvantage of this procedure was the loss of resolution caused by averaging relatively large groups of fractions. In spite of this limitation, however, it was still apparent that the MPO and lactoferrin sedimented at different densities. Nonspecific peroxidase sedimented with the MPO as did part of the lysozyme, a marker for both the specific and azurophil granules. The specific granule peak for lysozyme did not resolve sharply, probably due to the pooling of the fractions, but the azurophil peak was well resolved and it coincided with the MPO peak. Again the peak oxidase activities sedimented to a slightly greater density. These two experiments are representative of six separate experiments; in each separate experiment the peak of oxidase activities was found at a density greater than the MPO activity.
The oxidase activities were purified by this procedure. The total oxidase activity recovered from the gradient exceeds $100 \%$, suggesting removal of an inhibitor. In addition, the specific activity of the peak oxidase fraction in the gradients was 10-30 times that of the crude granule fraction.

\section{DISCUSSION}

In the present work, we have adapted a fluorometric procedure for the determination of pyridine nucleotides for use in measuring the reduced pyridine nucleotide oxidase(s) in human PMNL. This system is superior to the oxygen electrode or spectrophotometric methods presently in use in that it is at least 1,000 -fold more sensitive (9). It has several advantages over the isotopic system as well. It can measure both oxidase activities with as much sensitivity as the isotopic assay measures the NADPH oxidase. In addition, it is simpler and far less expensive to use than the isotopic procedure. We have applied this system to the study of the basic characteristics of the oxidases in granule fractions and have initiated purification of the enzyme(s) on sucrose density gradients.

Both oxidase activities were increased with phagocytosis, and both were insensitive to cyanide as evidenced by the fact that all assays were performed in $2.0 \mathrm{mM} \mathrm{KCN}$. Although both activities increased with increasing substrate concentration, time of incubation and protein, each may have had a slightly different $\mathrm{pH}$ optimum. Oxidation of NADPH was maximal at $\mathrm{pH} 5.5$ in agreement with previous reports (8), while oxidation of NADH appeared to be optimal at $\mathrm{pH}$ 6.0. Phagocytosis enhanced neither oxidase activity in granules isolated from CGD cells. Both activities sedimented to the same place in linear sucrose gradients.

A major question in the area of leukocyte metabolism is which enzyme (or more exactly which nucleotide oxidation) is responsible for the respiratory burst accompanying phagocytosis in human PMNL. The present work suggests that NADPH oxidation is likely to have more physiological significance than NADH oxidation. As illustrated in Figs. 3 and 6, the degree of NADPH oxidation was always greater than that of NADH when the substrate concentrations were equal (except at extremely high levels). In fact, in the range of $0.20-0.80 \mathrm{mM}$ the oxidation rate of NADPH was usually four to five times that of NADH. This was true every time the assay was performed, therefore, the procedure adopted for comparing the activities was to assay at $0.20 \mathrm{mM} \mathrm{NADPH}$ and $0.80 \mathrm{mM} \mathrm{NADH}$. Table I shows a compilation from several such experiments. In spite of the fact that the NADH concentration was four times that of $\mathrm{NADPH}$, in the phagocytizing samples there was 
significantly more oxidation of NADPH than NADH. This difference cannot be explained by a difference in protein because all assays were run at the same protein levels. Based on these data it seems reasonable to say that the oxidation of NADPH is responsible for the metabolic burst, since NADPH oxidation occurs at a lower concentration of substrate than does oxidation of NADH. This is particularly significant since the concentration of both reduced pyridine nucleotides within the human PMNL is estimated to be $0.05 \mathrm{mM}$ (19). In terms of the metabolic processes that occur in the cell after phagocytosis, NADPH fits into the scheme much more easily than does NADH. Oxidation of NADPH can directly explain the increase in oxygen consumption (as one of the reactants in the oxidase reaction), the increase in $\mathrm{H}_{2} \mathrm{O}_{2}$ production (as one of the products of the oxidase reaction) and the increase in the hexose monophosphate shunt activity (by providing NADP, the rate limiting substrate in the hexose monophosphate shunt activity).

Subcellular localization of the oxidase activity has been of some concern to workers in the field. Some investigators feel the plasma membrane is the site of this enzyme activity (20). Others have suggested that MPO would be a logical candidate for oxidase activity (21) since the oxidase behaves very much like a peroxidase and purified peroxidases are known to catalyze the oxidation of reduced pyridine nucleotides (22). Serious questions about the validity of these two hypotheses are raised by the sucrose gradient work presented here. Because there is no marker as yet for the membrane of human PMNL, it is impossible to argue absolutely against membrane localization, although the localization in the gradient would make this relatively unlikely.

The other possibility, then, is that the oxidase(s) are localized in the azurophil granule. Patriarca et al. (23) reported NADPH oxidase activity in the azurophil granule of rabbit PMNL, which seemed to support the hypothesis of Roberts and Quastel (21) that the oxidase was really MPO. The data here case some doubt on that possibility in human PMNL. Figs. 7 and 8 show that the peak activities of MPO and pyridine nucleotide oxidase did not co-sediment in a linear sucrose gradient. Although there was some overlap of the two activities, their peaks were distinctly separate. In Fig. 8 , the nonspecific peroxidase coincided with the immunologically determined MPO, and it too peaked before the oxidase. These data suggest that the oxidase is not MPO, nor does the oxidase seem to have peroxidative activity based on the o-dianisidine assay. MPO is found primarily in the lighter azurophil granule as reported by Spitznagel et al. (12), so if the oxidase peak is not coincident with the MPO peak, then it is not located in the light azurophil granule. Lysozyme is found in the specific granules and in the heavy azurophil granule (12). Fig. 8 shows the distribution of lysozyme in those particular gradients and it can be seen that the oxidase activities are located deeper in the gradient than lysozyme. Other gradients show this also. The data indicate that the oxidase activities are associated with very dense particulate material which sediments to $50-51 \%$ sucrose in $2 \mathrm{~h}$ time. The nature of the particle is presently unknown, but it does not seem to be the normal azurophil granule as it is presently known.

It is not possible to determine by these data whether there is one oxidase with different affinities for each nucleotide or two separate enzymes. The fact that the activities co-sediment in a linear sucrose gradient suggests, but does not prove, there is only one enzyme. In addition, the fact that in CGD both activities are defective further supports a one-enzyme hypothesis. But a final answer to this question will come only with the purification of the enzyme.

A very recent paper (24) described an NADH dependent nitro blue tetrazolium dye reductase with a very low $\mathrm{Km}$ for NADH which appeared to be absent in cells from patients with CGD. The relationship of this enzyme to the oxidase remains to be established; although we previously reported a low $\mathrm{Km}$ NADH diaphorase in human PMNL (19), we have been unable to detect oxidase activity toward low concentrations of NADH in either normal or CGD cells. Further, the location of the diaphorase in a sucrose density gradient (24) was quite different from that of the oxidase activities reported here. These discrepancies remain to be resolved.

Finally, in regard to purification of this enzyme, the work here shows the sucrose gradient centrifugation will be an invaluable tool in accomplishing this goal. Not only was more than $100 \%$ of the oxidase activity routinely recovered from the gradient, but the specific activity of the peak fractions in the gradient was 10-30 times that of a granule fraction prepared in parallel with the gradient. This will constitute the first major step in the purification of the enzyme(s). We are presently working toward that objective.

\section{ACKNOWLEDGMENTS}

We wish to thank Larry Martin for his excellent technical assistance. This research was supported by a grant from the Forsyth Cancer Service, by grant ER-40-1-3628, and by U. S. Public Health Service grants AI-10732, AI-02430, and CA-12197.

\section{REFERENCES}

1. Cagan, R. H., and M. L. Karnovsky. 1964. Enzymatic basis of the respiratory stimulation during phagocytosis. Nature (Lond.). 204: 255-257. 
2. Baehner, R. L., and M. L. Karnovsky. 1968. Deficiency of reduced nicotinamide-adenine dinucleotide oxidase in chronic granulomatous disease. Science (Wash. D. C.). 162: $1277-1279$.

3. Wilkinson, R. W., D. R. Powars, and P. Hochstein. 1975. New evidence for the role of NADH oxidase in phagocytosis by human granulocytes. Biochem. Med. 13: 83-88.

4. Patriarca, P., R. Cramer, S. Moncalvo, F. Rossi, and D. Romeo. 1971. Enzymatic basis of metabolic stimulation in leukocytes during phagocytosis: the role of activated NADPH oxidase. Arch. Biochem. Biophys. 145: 255-262.

5. Hohn, D. C., and R. I. Lehrer. 1975. NADPH oxidase deficiency in X-linked chronic granulomatous disease. J. Clin. Invest. 55: 707-713.

6. Evans, A. E., and N. O. Kaplan. 1966. Pyridine nucleotide transhydrogenase in normal human and leukemic leukocytes. J. Clin. Invest. 45: 1268-1272.

7. Reed, P. W. 1969. Glutathione and the hexose monophosphate shunt in phagocytizing and hydrogen peroxidetreated rat leukocytes. J. Biol. Chem. 244: 2459-2464.

8. DeChatelet, L. R., L. C. McPhail, D. Mullikin, and C. E. McCall. 1975. An isotopic assay for NADPH oxidase activity and some characteristics of the enzyme from human polymorphonuclear leukocytes. J. Clin. Invest. 55: 714-721.

9. Lowry, O. H., N. R. Roberts, and J. I. Kapphahn. 1957. The fluorometric measurement of pyridine nucleotides. J. Biol. Chem. 224: 1047-1064.

10. Kaplan, N. O., S. P. Colowick, and C. C. Barnes. 1951. Effect of alkali on diphosphopyridine nucleotide. J. Biol. Chem. 191: 461-472.

11. Lowry, O. H., N. J. Rosebrough, A. L. Farr, and R. J. Randall. 1951. Protein measurement with the Folin phenol reagent. J. Biol. Chem. 193: 265-275.

12. Spitznagel, J. K., F. G. Dalldorf, M. S. Leffel, J. D. Folds, I. R. H. Welch, M. H. Cooney, and L. E. Martin. 1974. Character of azurophil and specific granules purified from human polymorphonuclear leukocytes. Lab. Invest. 30: 774-785.

13. Böhlen, P., S. Stein, W. Dairman, and S. Udenfriend. 1973.
Fluorometric assay of proteins in the nanogram range. Arch. Biochem. Biophys. 155: 213-220.

14. Osserman, E. F., and D. P. Lawlor. 1966. Serum and urinary lysozyme (muramidase) in monocytic and monomyelocytic leukemia. J. Exp. Med. 124: 921-952 (and plates 91,92 ).

15. Worthington Biochemical Corporation. 1972. Worthington enzyme manual: peroxidase. Worthington Biochemical Corp., Freehold, N. J. 43.

16. Holmes, B., A. R. Page, and R. A. Good. 1967. Studies of the metabolic activity of leukocytes from patients with a genetic abnormality of phagocytic function. J. Clin. Invest. 46: 1422-1432.

17. McPhail, L. C., L. R. DeChatelet, and P. S. Shirley. 1975. NADPH oxidase activity of human neutrophils. J. Reticuloendothel. Soc. 18: 9b. (Abstr.)

18. Archer, G. T., and J. G. Hirsch. 1963. Isolation of granules from eosinophil leukocytes and study of their enzyme content. J. Exp. Med. 118: 277-286 (and plates 17-19).

19. DeChatelet, L. R., L. C. McPhail, D. Mullikin, and C. E. McCall. 1974. Reduced nicotinamide adenine dinucleotide and reduced nicotinamide adenine dinucleotide phosphate diaphorase activity in human polymorphonuclear leukocytes. Infect. Immun. 10: 528-534.

20. Briggs, R. T., D. B. Drath, M. L. Karnovsky, and M. J. Karnovsky. 1975. Localization of NADH oxidase on the surface of human polymorphonuclear leukocytes by a new cytochemical method. J. Cell. Biol. 67: 566-586.

21. Roberts, J., and J. H. Quastel. 1964. Oxidation of reduced triphosphopyridine nucleotide by guinea pig polymorphonuclear leukocytes. Nature (Lond.). 202: 85-86.

22. Akazawa, T., and E. E. Conn. 1958. The oxidation of reduced pyridine nucleotides by peroxidase. J. Biol. Chem. 232: 403-415.

23. Patriarca, P., R. Cramer, P. Dri, L. Fant, R. E. Basford, and F. Rossi. 1973. NADPH oxidizing activity in rabbit polymorphonuclear leukocytes: localization in azurophilic granules. Biochem. Biophys. Res. Commun. 53: 830-837.

24. Segal, A. W., and T. J. Peters. 1976. Characterization of the enzyme defect in chronic granulomatous disease. Lancet. I: 1363-1365. 